\title{
STATISTICAL ANALYSIS FOR IMPROVEMENT OF DOUBLE PERSISTENT SCATTERERS DETECTION IN SAR TOMOGRAPHY
}

\author{
Cosmin DĂNIȘOR ${ }^{1}$, Gianfranco FORNARO ${ }^{2}$, Antonio PAUCIULLO ${ }^{2}$, Mihai DATCU ${ }^{1,3}$ \\ ${ }^{1}$ University Politehnica of Bucharest, ${ }^{2}$ National Research Council of Italy, ${ }^{3}$ German Aerospace Center
}

\begin{abstract}
Synthetic Aperture Radar (SAR) tomography presents the advantage of multiple stable targets detection within same pixel. Fast-sup-GLRT (generalized likelihood ratio test based on support estimation) algorithm proved to be an ideal compromise between detection capabilities and computational complexity. In this work, a multi-look version of this detector which exploits the advantages of Capon estimation is examined. Statistical analysis of estimation and detection processes are conducted to compare the performances of sequential non-linear least-squares (NLLS) search and Capon filtering of projected data for double PS identification. Main objective is to exploit the super-resolution advantages of NLLS method without the risk of multiple stable targets classification from the same scattering contribution. For the last desiderate, an additional verification is included within the detection step.
\end{abstract}

Index Terms - SAR Tomography, fast-sup-GLRT, PS detection, Capon filtering, Non-Linear Lesast-Squares

\section{INTRODUCTION}

SAR tomography [1] is a multi-baseline technique which allows the reconstruction of scene's reflectivity profile in elevation direction, starting from a set of complex acquisitions. Its main advantage over classical interferometry [2] consists in the ability to separate the individual contributions of scatterers interfering within same range-azimuth resolution cell.

Reconstruction of reflectivity function's variation is conducted by means of spectral estimation techniques, starting from its available spectrum samples which can be retrieved from the complex images dataset. Main challenge of estimation process is posed by the fact that the available reflectivity spectrum samples are non-uniformly sampled, due to the irregular distribution of dataset's perpendicular baselines. A popular class of algorithms employed for reconstruction of scene's profile in elevation direction is given by the non-parametric spatial estimation methods, like Beam-Forming and Capon [3].

Generalized Likelihood Ratio Test (GLRT), which is equivalent to Beam-Forming filter's correlation index [4], proved an efficient method for detection of single persistent scatterers (PS) within the same image pixel. PS are targets with stable electromagnetic proprieties, being ideal for interferometric analysis due to their low temporal decorrelation. GLRT-based detection schemes for multiple PS are exploited in [5], by proposal of Sequential GLRT with cancellation (SGLRTC), and in [6] for construction of supGLRT method. Those detectors are based on the support estimation of reflectivity functions, capitalizing on their sparsity propriety given by the limited number of PS (two or more) present within same resolution cell. Sup-GLRT algorithm presents super- resolution capabilities, being able to detect scatterers situated below Rayleigh resolution, but this propriety comes with a computational cost. An adapted version of this detector, fast-supGLRT [7] carries a sequential estimation of signal's support. This adaptation is computationally efficient and introduces limited losses to detection capabilities.

In this paper, Capon estimation and fast-sup-GLRT detection are exploited to improve the process of single and double scatterers identification. Main objective's delineation is presented in section 3. Statistical analysis of estimation and detection processes are detailed in sections 4 and 5 , while the proposed solution for improvement of detection process is formulated in section 6 .

\section{SAR IMAGES DATASET}

The dataset used through this paper as a case study for the theoretical analysis consists of 32 single look complex images of Bucharest city, Romania, acquired by TerraSAR-X satellite in stripmap mode. Azimuth resolution of images is $3.3 \mathrm{~m}$, and ground range resolution equals $2 \mathrm{~m}$. Test region was selected around National Arena area, containing 800 azimuth lines and 1200 range samples.

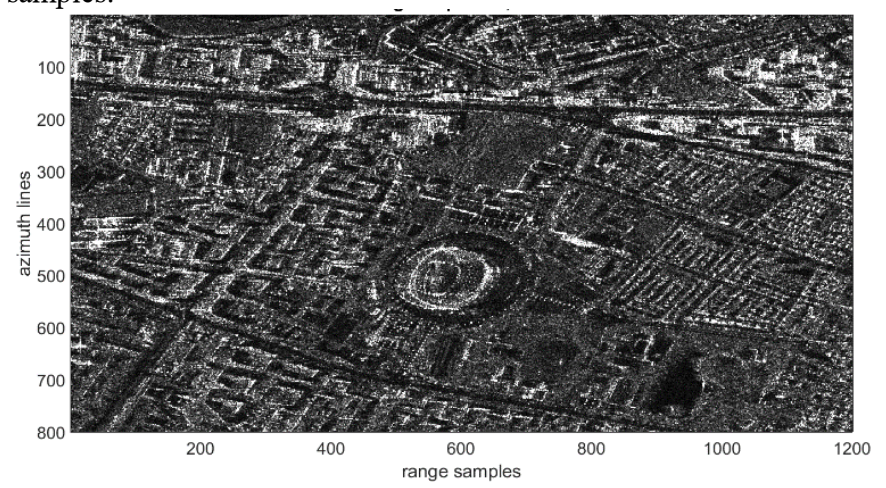

Figure 1 - Master image amplitude. Acquisition date: 20.04.2012

Test region's amplitude form the master image is presented in Figure 1. Dataset's perpendicular baselines range equals $431.97 \mathrm{~m}$, corresponding to a Rayleigh resolution in elevation [1] equal to $23.3 \mathrm{~m}$.

\section{PROBLEM FORMULATION}

For single and double PS detection, sup-GLRT algorithm is a twosteps test. First, data's total energy is compared to its part within the $\left(s_{1}, s_{2}\right)$ plane, defined by the (jointly) estimated elevation positions of primary and secondary scatterers under test. This step decides between the presence or absence of stable targets ( $H_{0}$ vs $\overline{H_{0}}$ hypothesis). If the presence of stable scatterers is declared, the data energy within the $\left(s_{1}, s_{2}\right)$ plane is compared to the one corresponding to the dominant scatterer's (singly) estimated 
direction $s_{1}$, to discriminate between the presence of one or two stable targets ( $H_{I}$ vs $H_{2}$ hypothesis):

$$
\begin{gathered}
1-\frac{\left|\boldsymbol{g}^{H} \boldsymbol{P}_{s_{1} s_{2}}^{\perp} \boldsymbol{g}\right|}{\boldsymbol{g}^{H} \boldsymbol{g}} \stackrel{\substack{\bar{H}_{0} \\
>}}{>} T_{1} \\
1-\frac{\left|\boldsymbol{g}^{H} \boldsymbol{P}_{s_{1} s_{2}}^{\perp} \boldsymbol{g}\right|}{\left|\boldsymbol{g}^{H} \boldsymbol{P}_{s_{1}}^{\perp} \boldsymbol{g}\right|} \stackrel{\stackrel{H_{2}}{>}}{\underset{H_{1}}{>}} T_{2}
\end{gathered}
$$

where $\boldsymbol{g}$ is the data vector and $P^{\perp}$ are the projectors orthogonal to $s_{1}$ direction, respectively to $\left(s_{\left.1, s_{2}\right)}\right.$ plane. The detection scheme follows a CFAR approach, therefore thresholds $T_{i}$ can be set according to the desired false alarm probability.

In [6] the direction $s_{1}$ and the plane $\left(s_{1}, s_{2}\right)$ are estimated by mean of a 1D and a 2D Non Linear Least Square (NLLS) search, respectively. It is worth pointing out that the 1D-NLLS is equivalent to the Beam Forming filtering. Considering that two dimensional search is computationally demanding, fast-sup-GLRT algorithm proposes a sequential estimation of target's positions: $s_{1}$ position is fixed to the one identified by the 1D-NLLS search, thus reducing also the 2D-NLLS operation to a $1 \mathrm{D}$ search. No limit is imposed between the positions of dominant and secondary scatterers in original versions of sup-GLRT and fast-sup-GLRT algorithm [6]-[7]

A multi-look (ML) version of fast-sup-GLRT detector is proposed and implemented in this work. To mitigate noise effects, the detection algorithm can be combined with DespeKS method presented in [8]. ML elements are chosen from a broader window, considering only the points whose data vector's amplitude has a similar distribution with the one from window's central point. Kolmogorov-Smirnov test will be applied along a 9x9 window for identification of statistical homogenous points.

Instead of 1D-NLLS search, Capon filtering will be implemented for estimation of dominant targets position. Elevation $s_{l}$ is identified as the coordinate of Capon filter's maximum output power $P_{c}$ :

$$
\max _{s} P_{c}=\max _{s} \frac{1}{\boldsymbol{a}(s)^{H} \boldsymbol{R}_{g}^{-1} \boldsymbol{a}(s)}
$$

where $\boldsymbol{R}_{g}$ is data's covariance matrix (estimated with the adaptive ML algorithm), and $\boldsymbol{a}(s)$ is the steering vector. This adaptation is considered because Capon filter presents better side-lobs attenuation than Beam-Forming (1D NLLS search), being more suitable for reflectivity estimation process. For secondary scatterer's elevation identification, Capon filtering can only be applied on data from which dominant target's contribution has been subtracted. Data's projection can be implemented directly at covariance matrix level:

$$
\boldsymbol{R}_{\boldsymbol{g} \mathbf{2}}=\left(\boldsymbol{I}-\boldsymbol{a}\left(s_{1}\right) \boldsymbol{a}\left(s_{1}\right)^{H}\right) \boldsymbol{R}_{\boldsymbol{g}}\left(\boldsymbol{I}-\boldsymbol{a}\left(s_{1}\right) \boldsymbol{a}\left(s_{1}\right)^{H}\right)
$$

where $\boldsymbol{R}_{g 2}$ is the covariance matrix of the projected data and $\boldsymbol{I}$ denotes the identity matrix. Despite its estimation advantages, this method does not have super-resolution capabilities. Because of data's projection, scatterers located closer to Rayleigh resolution limit cannot be detected. By contrast, in case of NLLS search, which can identify closely positioned scatterers, it appears the risk that a single target contribution will be wrongly classified as both primary and secondary PS.

Those situations are exemplified and analyzed below. In the presented situations, Capon estimation is applied to identify dominant target's position sl. For determination of secondary scatterers elevation, performances of Capon filtering on projected data and sequential NLLS search (with fixed $s_{1}$ position) are compared. Elevation pair $\left(S_{1}, S_{1}\right)$ is excluded from the NLLS exploration. The search grids of $s_{1}$ and $s_{2}$ positions are defined between -60 and $60 \mathrm{~m}$ with $0.5 \mathrm{~m}$ sampling rate.
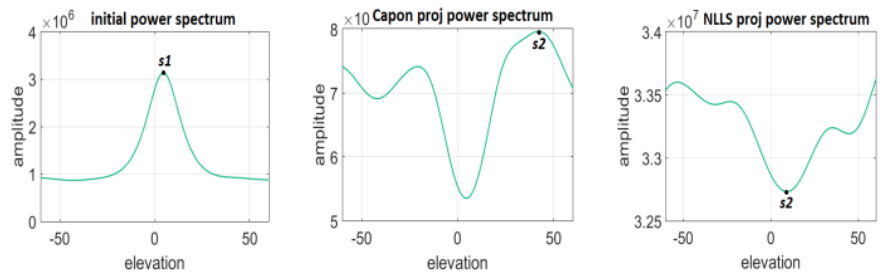

Figure 2 - Initial estimated power spectrum (left), Capon spectrum of projected data (center), and NLLS projector's variation after $s_{2}$ (right), resolution cell with a single scatterer

From the initial estimated reflectivity function, it can be observed that the resolution cell analyzed in Figure 2 contains a single scatterer $\left(s_{I}=4.5 \mathrm{~m}\right)$. As expected, Capon spectrum of the projected data presents its minimum value in the region of dominant target position $s_{1}$. Same observation is valid in case of absolute variation after $s_{2}$ of NLLS projector $\boldsymbol{g}^{H} \boldsymbol{P}_{\varepsilon_{1} \varepsilon_{2}}^{\perp} \boldsymbol{g}$ ( $s_{1}$ fixed), both projected reflectivity functions (Capon and 2D-NLLS) being similar. Main difference is that, in case of projected data's Capon spectrum, $s_{2}$ elevation is identified in the position of function's maximum value $\left(s_{2}=42 \mathrm{~m}\right)$, while in case of NLLS search, $s_{2}$ position is chosen in the minimum point of the projection $\left(s_{2}=6.5 \mathrm{~m}\right)$. Therefore, in the last case, $s_{2}$ value is identified close to $s_{1}$ position, leading to the risk that the contribution of the same target will be tested for both dominant and secondary PS classification. In this situation, Capon reflectivity estimation from the projected data has an advantage.
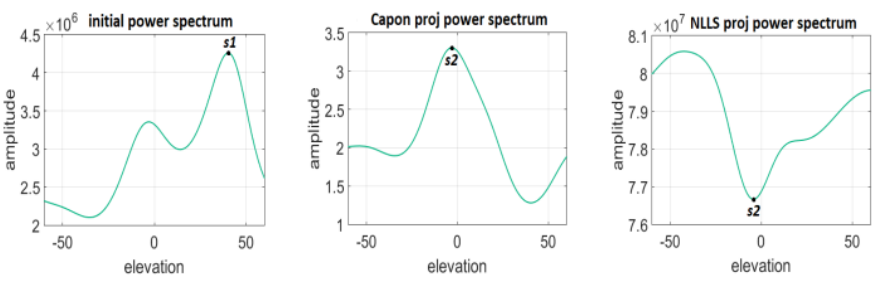

Figure 3 - Initial estimated power spectrum (left), Capon spectrum of projected data (center), and NLLS projector's variation after $s_{2}$ (right), pixel with two scatterers located above Rayleigh resolution

Initial data's Capon spectrum presented in Figure 3 indicates the case of a resolution cell which contains two distinct scatterers $\left(s_{1}=40.5 \mathrm{~m}\right)$, located at a distance greater than the Rayleigh resolution. In this case, positions of Capon projected spectrum's maximum value and NLLS projector's minimum value are close, both methods generating similar results $\left(s_{2 C}=-3.5 \mathrm{~m}, s_{2 N}=-4.5 \mathrm{~m}\right)$.

Presence of two closely located scatterers can be observed in the originally estimated Capon spectrum presented in Figure 4 $\left(s_{l}=41 \mathrm{~m}\right)$. Targets are located below Rayleigh resolution, and secondary scatterer's contribution, though observable, does not present a distinct peak. Carrying the aforementioned principle for identification of $s_{2}$ elevation, it can be observed that NLLS projection identifies the realistic position of secondary target $\left(s_{2 N}=20 \mathrm{~m}\right)$, closer to the dominant one, while Capon projection doesn't have the capability to cut beyond Rayleigh resolution 
$\left(s_{2 C}=16 m\right)$. Therefore, in this situation, NLLS search presents an advantage.
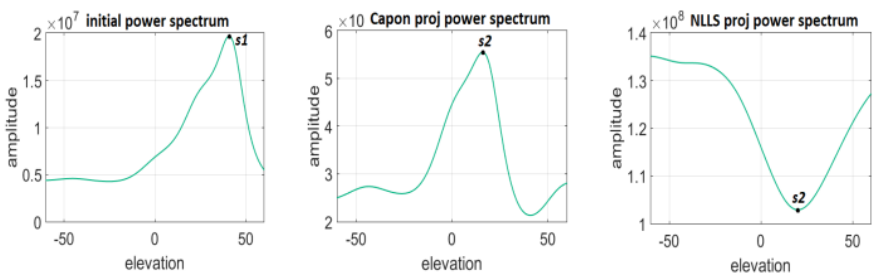

Figure 4 - Initial estimated power spectrum (left), Capon spectrum of projected data (center), and NLLS projector's variation after $s_{2}$

(right), pixel with two scatterers located beyond Rayleigh res.

Main objective of this work is to find an adaptation of fast-supGLRT detector, which exploits the advantages of Capon filtering for estimation process and super-resolution capabilities of NLLS search, avoiding multiple PS detection from the same target's contribution.

\section{ELEVATION ESTIMATION STATISTICS}

A comparative study between estimation of secondary scatterers elevation using NLLS search and Capon filtering of projected data is presented. For NLLS algorithm, two situations are analyzed: between elevation of primary and secondary scatterers from within same resolution cell no limits were set in first instance, then a minimum distance equal to $1 / 3$ of Rayleigh resolution was imposed. In all of the three analyzed algorithms, dominant targets elevation $s_{1}$ is estimated with Capon method. Then, sequential NLLS search over $s_{2}$ or Capon filtering on projections are applied.
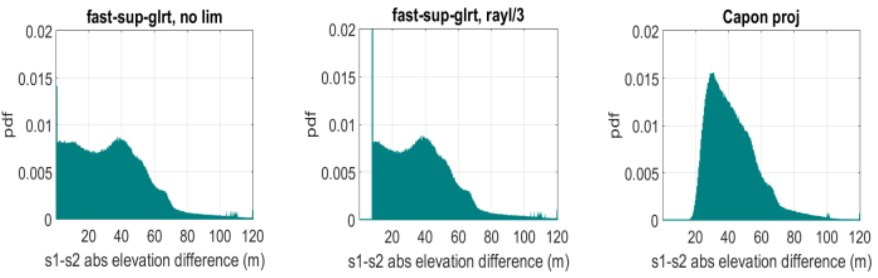

Figure 5 - Normalized histograms of differences between elevations of primary and secondary scatterers, NLLS (left), NLLS with one third of Rayleigh resolution $s_{1}-s_{2}$ limit (center) and Capon

filtering of projected data (right) for estimation of secondary targets elevation

Histograms of differences between elevation of primary and secondary targets from the same resolution cell, estimated with the three methods under test, are presented in Figure 5. In case of unrestricted NLLS search, number of points with minimum possible $s_{1}-S_{2}$ difference (elevation sampling rate) is considerably higher than the rest of the distribution values: the histograms presents 240 samples, and $1.4 \%$ of scene's points are located at minimum $0.5 \mathrm{~m}$ elevation difference. For the NLLS implementation were mandatory distance of one third of Rayleigh resolution was imposed, the percent of pixels whose targets are located at the minimum possible distance of $7.5 \mathrm{~m}$ is even higher: $12.98 \%$. This percent is equal to the one of points identified at a distance lower than Rayleigh resolution per three in the unrestricted NLLS search. Above the Rayleigh per three value, both unrestricted and restricted NLLS histograms are identical. Those facts confirm that the imposed limit of one third of Rayleigh resolution was identified as the elevation distance between all the points which were detected as located at a lower distance in the unrestricted NLLS search, before imposing the limit. This is a consequence of the fact that the width of lower main lobe of NLLS variation after $s_{2}$ is close to Rayleigh value. Therefore, in case of points whose secondary elevation was identified close to the primary one, minimum value of the projector will be identified still across the main lower lobe, at the limit of the restricted search interval, so the absolute $s_{1-S 2}$ elevation difference of those points will equal one third of Rayleigh resolution. As expected, absolute elevation differences do not go beyond Rayleigh limit in case of Capon filtering of projected data, as it can be noticed from the corresponding histogram.

Therefore, the limit imposed during the estimation process between double scatterers elevations leads to artificial results. The described phenomenon appeared also when imposing a NLLS search limit equal to half of Rayleigh resolution, $18.14 \%$ of scene's pixels containing targets identified as located at the increased limit. A further increase of the minimum imposed distance between primary and secondary targets isn't reasonable. Setting this limit close to Rayleigh resolution means that NLLS search loses its super-resolution advantages over Capon filtering of projected data.

\section{PS DETECTION STATISTICS}

Fast-sup-GLRT detector was implemented for the three estimated elevation datasets analyzed in the previous section. Statistics (number and percent from total resolution cells of the scene) of single and double stable targets detected in the three situations are synthetized in Table 1.

\begin{tabular}{|c|c|c|c|c|}
\hline \multirow{2}{*}{} & \multicolumn{2}{|c|}{ single PS } & \multicolumn{2}{c|}{ double PS } \\
\cline { 2 - 5 } & number & percent & number & percent \\
\hline $\begin{array}{c}\text { NLLS } \\
\text { no limit }\end{array}$ & 268861 & $28 \%$ & 28046 & $2.92 \%$ \\
\hline $\begin{array}{c}\text { NLLS } \\
\text { RI/3 limit }\end{array}$ & 268748 & $27.99 \%$ & 27750 & $2.89 \%$ \\
\hline $\begin{array}{c}\text { Capon } \\
\text { proj data }\end{array}$ & 261924 & $27.28 \%$ & 20661 & $2.15 \%$ \\
\hline
\end{tabular}

Table 1 - Statistics of detected single and double PS
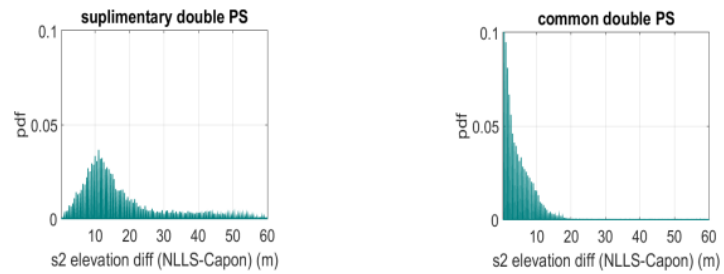

Figure 6 - Normalized histograms of differences between NLLS and Capon estimations of secondary scatterers elevation, for double PS detected exclusively by NLLS search (left), and double

PS detected also by Capon estimation of $s_{2}$ (right)

The additional imposing of minimum distance equal to one third of Rayleigh resolution between double scatterers elevation does not lead to a significant drop of the number of detected PS. The major difference appears between NLLS and Capon estimation of secondary scatterers elevation: in case of Capon filtering of projected data, the number of detected double stable targets decreases with $26.33 \%$ compared to the case of NLLS estimation. All of the double PS detected by Capon estimation of $s_{2}$ elevation are also identified in the sequential NLLS search method. A significant $89.88 \%$ of double PS detected exclusively by NLLS search present an absolute $s_{1-s_{2}}$ elevation difference below 
Rayleigh resolution Figure 6 presents the histograms of differences between NLLS and Capon estimates of secondary scatterers elevation $s_{2}$, in set of cells commonly classified as double PS and in points were only NLLS search identified the presence of double stable targets. Unsurprisingly, distribution of $s_{2}$ elevation differences presents a higher mean and dispersion in secondary PS points detected exclusively by NLLS search $(\mu=17.91 \mathrm{~m}$ and $\sigma^{2}=181.1 \mathrm{~m}^{2}$ ), compared to the statistics of same distribution in points classified as double PS by both NLLS and Capon based methods $\left(\mu=4.65 m\right.$ and $\left.\sigma^{2}=26.36 m^{2}\right)$.

\section{PROPOSED SOLUTION}

Super-resolution advantage of NLLS search is confirmed by the supplementary detected double PS. The objective is to exploit this advantage, but eliminating the secondary PS identified from the same scattering contribution as the dominant ones. This elimination process didn't generate the expected results when applied during the reflectivity estimation step, by imposing minimum $s_{1}-S_{2}$ distance, therefore it will be implemented after the detection process. Single PS detection isn't greatly influenced by the secondary scatterer elevation estimation method. Double PS detected by Capon filtering of projected data will not be altered, since their presence was also confirmed by sequential NLLS search method. In case of supplementary double PS detected exclusively by NLLS algorithm, those who present an elevation difference between the two targets lower than one third of Rayleigh resolution will be eliminated, since it is plausible that multiple detection of same scattering contribution occurred.

\section{RESULTS AND CONCLUSIONS}

After implementation of the proposed refinement process, $33.68 \%$ of double PS detected exclusively by NLLS search were eliminated. Total number of detected double scatterers becomes equal to 25558 , which represents $2.58 \%$ of scene's resolution cells, leading to an increase with $23.7 \%$ relative to the number of double PS identified by Capon filtering of projected data.

Spatial distributions of single stable targets (identified with NLLS search process of $s_{2}$ value) and the refined set of double PS are presented in Figure 7. Associated elevation values are estimated with Capon filtering in case of single PS and with sequential NLLS search in case of double PS. As expected, stable targets are mainly identified on roof and facades of constructions. Double PS are identified in regions prone to layover phenomenon occurrence.

Main contributions presented in this work consist in adaptation of fast-sup-GLRT detector to a ML version which uses Capon filtering for dominant scatterers position estimation and implementation of a statistical analysis to determine an optimal procedure which avoids multiple PS classification of same scattering contribution. This supplementary analysis is required because the multi-look leads to a widening of the main lobe of scatterer's response, since contributions present in multiple resolution cells are averaged. Therefore, in case of the presence of a single scatterer, NLLS is prone to estimate the position of the secondary scatterer close to the one of the primary. A minimum distance equal to one third of Rayleigh resolution was imposed between double scatterers elevation positions. The analysis indicated that the optimal way to impose this constraint is within the detection process, in points classified exclusively by NLLS search as double PS, since those are the ones placed within a distance below Rayleigh resolution.
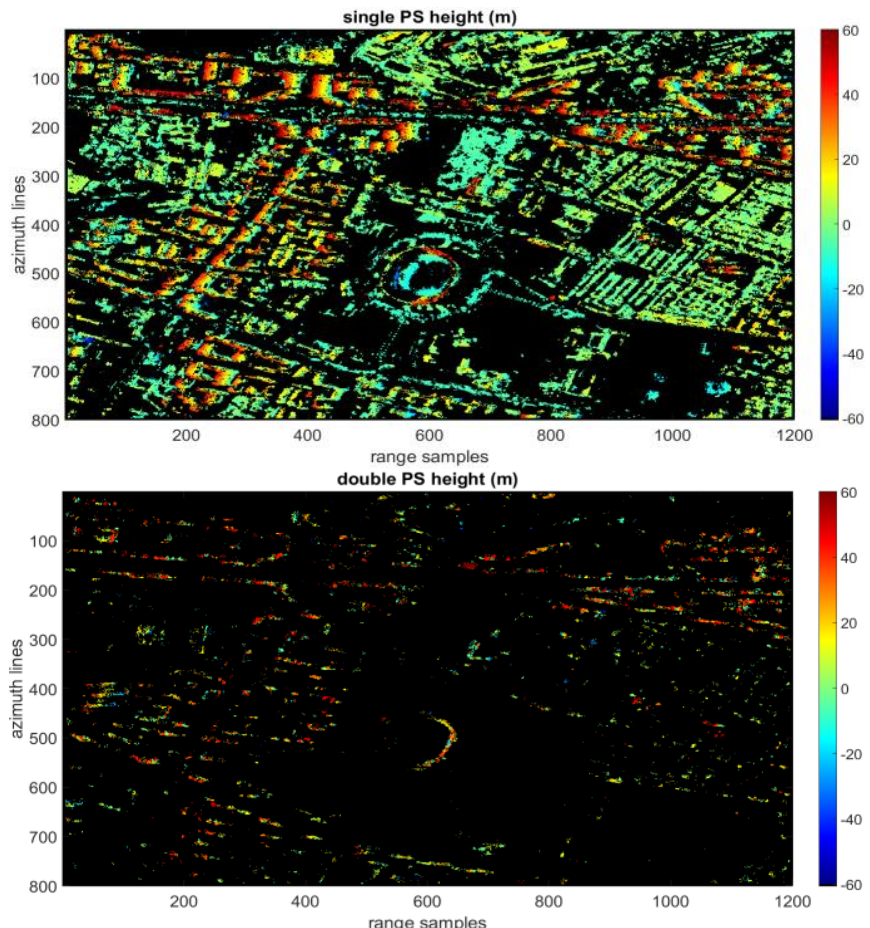

Figure 7 - Spatial distribution and estimated elevation values of detected single PS (up) and double PS, upper layer (down)

For future work, the presented analysis will be extended to $5 \mathrm{D}$ space, by inclusion of linear deformation rates and thermal dilation dimensions.

\section{REFERENCES}

[1] G. Fornaro , F. Serafino and F. Soldovieri, "Three-dimensional focusing with multipass SAR data," IEEE Transactions on Geoscience and Remote Sensing, vol. 41 pp. 507 - 517 , 2003.

[2] R. Bamler and P. Hartl, "Synthetic Aperture Radar Interferometry," Inverse problems, vol. 14, pp. R1 - R54, 1998.

[3] C. Danisor, G. Fornaro and M. Datcu, "Comparative study of SAR tomographic reconstruction algorithms," in Telecommunications Forum (TELFOR), Belgrade, 2015.

[4] A. De Maio, G. Fornaro and A. Pauciullo, "Detection of Single Scatterers in Multidimensional SAR Imaging," IEEE Transactions on Geoscience and Remote Sensing, vol. 47, pp. 2284 - 2297, 2009

[5] A. Pauciullo, D. Reale, A. De Maio and G. Fornaro, "Detection of Double Scatterers in SAR Tomography," IEEE Transactions on Geoscience and Remote Sensing, vol. 50, pp. 3567 - 3586, 2012.

[6] A. Budillon and G. Schirinzi, "GLRT Based on Support Estimation for Multiple Scatterers Detection in SAR Tomography," IEEE JSTARS, vol. 9, pp. 1086-1094, 2016.

[7] A. Budillon, A. Johnsy and G. Schirinzi, "A Fast Support Detector for Superresolution Localization of Multiple Scatterers in SAR Tomography" IEEE JSTARS, vol. 10, pp. 2768 - 2779 , 2017.

[8] A. Ferretti , A. Fumagalli , F. Novali , C. Prati , F. Rocca and A. Rucci , "A New Algorithm for Processing Interferometric DataStacks: SqueeSAR," IEEE Transactions on Geoscience and Remote Sensing, vol. 49, no. 9, pp. 3460 - 3470 , 2011. 